\title{
ULTRABORNOLOGICAL STRUCTURES
}

\author{
V. MURALI \\ Department of Mathematics \\ Rhodes University \\ P 0 Box 94 \\ Grahamstown \\ 6140 \\ SOUTH AFRICA
}

(Received October 24, 1989 and in revised form November 30, 1990)

\begin{abstract}
The purpose of this note is to extend Warner's idea of "bornological structures" to cover non-locally convex situations and to develop a framework unifying several variations on an ultrabornological theme ( for example, ultrabornological spaces, 0-ultrabornological spaces, i-ultrabornological algebras). We do this by first generalising the concept of a "structure" on a vector space as defined by Warner.
\end{abstract}

Key words and phrases: structure, B-bornivorous, B-ultrabornological, structured *-inductive limits, convex structures, general structures.

AMS(MOS) subject classification: Primary 46A09, 46Al5, Secondary $46 \mathrm{~A} 40,46 \mathrm{AO} 5$.

\section{INTRODUCTION}

In [10], Warner developed a framework with which various "bornological concepts" can be unified and properties of the several variations on a bornological theme (for example, bornological spaces, o-bornological spaces, 1 bornological algebras) may be derived from results established within this general framework. Recently, these variations have been extended in the setting of non-locally convex spaces and algebras (see, for example [3]. [7], [8]). In this paper, we have sought to extend Warner's ideas to cover the non-locally convex situations. In section 2, we have generalised the concept of a "structure" on a vector space as defined by Warner in [10] and have given some basic properties of B-ultrabornological structured spaces. In Section 3, we briefly discuss the structured *-inductive limits of a family of structured topological vector spaces. Finally, we give examples of various structures both convex and non-convex, and have been able to fit into our general framework ultrabornological spaces, o-ultrabornological spaces and i-ultrabornological algebras. 


\section{STRUCTURED SPACES}

DEFINITION 2.1 Let $E$ be a vector space and let $\boldsymbol{V}$ be a collection of balanced subsets of $E$. Then $V$ is said to be a structure on $E$ if the following conditions are satisfied.

(i) $V$ is a filter base on $E$.

(ii) $E,\{0\} \in V$.

(iii) For each $A \in V$, there exists a sequence $\left\{A_{n}\right\}(n=1,2 \ldots)$ in $V$ such that $A_{1}+A_{1} \leq A$,

$A_{n+1} A_{n+1} \leq A_{n}(n=1,2 \ldots)$. (The sequence $\left\{A_{n}\right\}$ is called a defining sequence for $A$ ).

The above generalises the concept of a structure on a vector space as defined by Warner in [10]. Every suprabarrel defines a structure on a vector space; for if $V$ is a suprabarrel in a vector space $E$, with a defining sequence $\left\{V_{n}\right\}$, then the collection $\boldsymbol{V}=\left\{\mathrm{V},\{0\}, E, V_{n}(n=1,2, \ldots)\right\}$ defines a structure on $E$. Thus, the above definition generalises the notion of a suprabarrel in a vector space.

DEFINITION 2.2 A structured space is a pair (E, $V$ where $V$ is a structure on $E$.

DEFINITION 2.3 A linear topology $\tau$ on a structured space $(E, V$ is said to be compatible with $\boldsymbol{V}$, if $\boldsymbol{V}$ contains a base of $\boldsymbol{\tau}$-neighbourhoods of the origin.

A structured topological vector space is a triple (E, $V, \tau)$ where (E, $V$ is a structured space and $\tau$ is a linear topology on $E$ compatible with $\nabla$.

Every linear topology $\tau$ on a vector space $E$, with a $\tau$-neighbourhood base $\boldsymbol{U}$ at the origin, gives rise to a structure $\nabla_{\tau}$ on $E$, with which it is compatible. $V_{\tau}$ will consist of $E,\{0\}, \sigma$, and $\tau$ is the finest linear topology on $E$ compatible with $\nabla_{\tau} . \quad \nabla_{\tau}$ is called the structure associated with $\tau$. Conversely, there is always at least one linear topology on $E$ compatible with any given structure, namely, the topology whose only open sets are $E$ and $\phi$. . When no confusion arises, a structured topological vector space (E, $V, \tau$ ) is denoted simply by $E$.

DEFINITION 2.4 If (E, $D$ ) and (F, $H$ ) are structured spaces, then a mapping $f$ of $E$ into $F$ is said to be a structured homomorphism is $f$ is a linear mapping, and $f(V) \subseteq W$ and $f^{-1}(W) \subseteq V$.

Clearly, the composition of two structured homomorphisms is a structured homomorphism. If $f$ is a linear mapping from a structured space ( $E$, $V$ ) onto a vector space $F$, then $\{F, f(V)\}$ is a structured space. Similarly, if $f$ is a linear mapping from a vector space $E$ onto a structured vector space $(F$, $W$, then $f^{-1}(n)$ is a structure on $E$.

Let (E, $V$ be a structured space and suppose that $B$ is a collection of subsets of $E$. 
DEFINITION 2.5 We say that a subset $\mathrm{V}$ of $\mathrm{E}$ is $\underline{B}$-bornivorous if $\mathrm{V} \boldsymbol{\nabla}$ and has a defining sequence $\left\{V_{n}\right\}$ in $V$ such that each $V_{n}$ is absorbing and also absorbs each $B \in B$.

DEFINITION 2.6 Let $(E, V, \tau)$ be a structured topological vector space. Then $E$ is said to be a B-ultrabornological structured space if each member of $B$ is $\tau$-bounded and every $B$-bornivorous subset of $E$ is a $\tau$-neighbourhood of the origin.

We note that if $(E, V, \tau)$ is $B$-ultrabornological and if $W$ is a structure on E weaker than $\boldsymbol{V},(i . e$.$) if \boldsymbol{W} \subseteq \boldsymbol{V}$, but with which $\tau$ is compatible, then $(E, \boldsymbol{W} \tau)$ is also B-ultrabornological. In the following we define two concepts connecting a linear mapping and the class of subsets $B$.

DEFINITION 2.7 Let $(F, \eta)$ be a topological vector space. A linear mapping $f$ from $E$ into $F$ is said to be $B$-ultrabornological if, for every $\eta$-neighbourhood $W$ of 0 in $F, f^{-1}(W)$ contains a $B$-bornivorous subset.

We now consider some general properties of B-ultrabornological structured spaces.

PROPOSITION 2.8 Let $(E, \tau)$ and $(F, \eta)$ be two structured topological vector spaces, $f$ a linear mapping from $E$ into $F$, and $B$ a class of $\tau$-bounded subsets of $E$. If $f$ is continuous, then $f$ is $B$-ultrabornological, and if $f$ is $B$-ultrabornological, then $f$ is $B$-bounded. If $f$ is a structured homomorphism, then $f$ is $B$-ultrabornological if and only if $f$ is $B$-bounded.

PROOF. If $V$ is a $\eta$-neighbourhood of 0 in $F, f^{-1}(V)$ is a $\tau$-neighbourhood of $O$ in $E$, and so a B-bornivorous subset. Hence $f$ is B-ultrabornological.

Secondly, if $W$ is a $\eta$-neighbourhood of $O$ in $F$, then, since $f$ is $B$ ultrabornological, $f^{-1}(W) \geq V$, where $V$ is a $B$-bornivorous subset of $B$. Thus, for each $B \in B$, there exists a $\lambda>0$ such that $\lambda B \subseteq V \subseteq f^{-1}(W)$; that is, $f(B) \subseteq 1 / \lambda$ $W$ which implies that $f(B)$ is $\eta$-bounded. Thus $f$ is $B$-bounded. The last part of the Proposition is straightforward.

THEOREM 2.9 Let $(E, V, \tau)$ be a structured topological vector space and $B$ a class of $\tau$-bounded subsets of $E$. Then the class of all B-bornivorous subsets of $E$ is a base of neighbourhoods of the origin for a linear topology $\tau *$ on $E$ compatible with $\nabla$. $\tau^{*}$ is finer than $\tau$ and each $B \in B$ is $\tau^{*}$-bounded. (E, $\boldsymbol{V}, \tau$ ) is B-ultrabornological if and only if $\tau=\tau *$. Also, if $f$ is a structured homomorphism from $E$ into any structured topological vector space $F$, then $f$ is $B$ bounded from $(E, \tau)$ into $F$ if and only if $f$ is continuous from $\left(E, \tau^{*}\right)$ into $F$. PROOF. Clearly the family $\sigma$ of all $B$-bornivorous subsets $\left\{U_{1}\right\}(i \in I)$ is a filter base on $E$ such that $\boldsymbol{V}$ contains $U$. Also, be definition, each $U_{1} \in U$ is a suprabarrel. Therefore, by Proposition 2.2 of [6] $\boldsymbol{U}$ is a base of neighbourhoods of 0 for a linear topology $\tau^{*}$ on $E$ and it is clearly compatible with $V$. Since each $\tau$-neighbourhood is a $B$-bornivorous subset, $\tau$ is coarser than 
$\tau^{*}$. Moreover, every $B$-bornivorous subset absorbs each $B \in B$ implying that elements of $B$ are $\tau *$-bounded. It is evident by Definition 2.6 , that $(E, V, \tau$ ) is B-ultrabornological if and only if $\tau=\tau^{*}$. The last assertion follows easily from Proposition 2.8

THEOREM 2.10 Let $(E, V, \tau)$ be a structured topological vector space and $B$ a class of $\tau$-bounded subsets of $E$. Then the following conditions are equivalent.

(i) E is a B-ultrabornological structured space.

(ii) Every B-ultrabornological linear mapping from $E$ into any topological vector space is continuous.

(iii) Every B-bounded structured homomorphism from $E$ into any structured topological vector space is continuous.

(iv) If $\tau^{1}$ is any topology on $E$ compatible with $\boldsymbol{V}$ such that the identity mapping from $(E, \tau)$ onto $\left(E, \tau^{1}\right)$ is B-bounded, then it is continuous.

(v) $\tau$ is finest of those topologies on $E$ compatible with $\boldsymbol{V}$ and for which each $B \in B$ is bounded.

PROOF. (i) - (ii). Since $E$ is a B-ultrabornological structured space, every B-bornivorous subset of $\mathrm{E}$ is a $\tau$-neighbourhood of the origin and so implies (ii).

(ii) - (iii). This follows from Proposition 2.7.

(iii) - (iv). This is trivial.

(iv) $\rightarrow(v)$. If $\tau^{1}$ is topology on $E$ compatible with $\nabla$ and for which each $B \in B$ is $\tau^{1}$-bounded, then the identity mapping from $(E, \tau)$ onto $\left(E, \tau^{1}\right)$ is $B$ bounded and thus continuous by hypothesis. Hence $\tau$ is finer than $\tau^{1}$.

(v) - (i). By Theorem 2.8, $\tau *$ is compatible with $V$ and each $B \in B$ is bounded for $\tau^{*}$. Hence as $\tau$ is coarser than $\tau^{*}$, by hypotheses $\tau=\tau *$ and so $E$ is a $B$ ultrabornological structured space.

CORALlaRY. Let (E, $\nabla, \tau$ ) be à structured topological vector space and $B$, a class of $\tau$-bounded subsets of $E$. Then $E$ is $B$-ultrabornological if every $B$ bounded linear mapping into any structured topological vector space is continuous and conversely.

PROOF. If $\mathrm{E}$ is B-ultrabornological, then since (i) $\rightarrow$ (iii), it follows that every $B$-bounded linear mapping into any structured topological vector space is continuous. Conversely, if every B-bounded linear mapping into any structured topological vector space is continuous, then in particular the identity mapping is continuous and so (iv) implies (i), as required.

We conclude this section with some remarks on the quotient and product structures.

REMARK (i). Let ( $E, V$ be a structured space, $F$ a subspace of $E$ and $\phi$ 
the canonical mapping $\phi: E \rightarrow E / F$. Since $\phi$ is a onto linear mapping, by the remark made after Definition $2.3, \phi(V)$ is a structure on E/F. If $\tau$ is a topology on $E$ compatible with $\boldsymbol{V}$, then it is easy to see that the quotient topology, $\phi(\tau)$ say, on $E / F$ is compatible with $\phi(V) . \phi(V)$ is called the quotient structure on E/F.

REMARK ( $i i)$. Let $\left(E_{i}, \nabla_{i}, \tau_{i}\right)$ ( $\left.i \in I\right)$ be a family of structured topological vector spaces and $E$ be the product space $I I E_{1}$, and $\tau$ be the product topology on E. For each $i \in I$, let $J_{1}$ denote the injection mapping from $E_{i}$ into E. Consider the collection $\boldsymbol{V}$ of all balanced subsets $V_{0}$ of $E$ such that $V_{0}$ has a defining sequence of balanced subsets $\left\{V_{n}\right\}(n=1,2, \ldots)$ in $E$ with the property that $J_{1}^{-1}\left(V_{n}\right) \in V_{1}$ for each $i \in I$ and $n=0,1,2, \ldots$ Then it is not difficult to show that $\boldsymbol{V}$ is a structure of $E$ and that $\tau$ is compatible with $\boldsymbol{V}$. $\boldsymbol{V}$ is called the product structure on $E$.

\section{STRUCTURE *-INDUCTIVE LIMITS}

In this section, we consider the *-inductive limits of a family of structured topological vector spaces. In detail, we have the following situation. Let ( $E_{1}$,

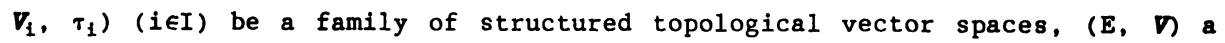
structured space, and, for each $i \in I$, suppose that $g_{1}$ is a structured homomorphism from $E_{1}$ into $E$ such that $E=U g_{i}\left(E_{1}\right)$. The set $\Phi$ of all topologies on $E$ compatible with $\boldsymbol{V}$ relative to which each $g_{1}$ is continuous is not empty, since it contains the trivial topology. Hence there is a finest such topology $\tau$ on $E$.

DEFINITION 3.1 The topology $\tau$ as defined above is called the structured *-inductive limit topology on (E, V) relative to the structured topological vector spaces $\left\{E_{1}\right\}$ and the structured homomorphisms $\left\{g_{1}\right\}(i \in I) ;(E, \nabla, \tau)$ is called the structured *-inductive limit of $\left(E_{i}, V_{i}, \tau_{1}\right)$ with respect to $g_{i}$.

EXAMPLE 1. Let $(E, V, \tau)$ be a structured topological vector space, $\mathbf{F}$ a subspace of $E$ and $\Phi$ the canonical mapping of $E$ onto $E / F$. Let $\Phi(V)$ and $\Phi(\tau)$ be the quotient structure and quotient topology on $E / F$ respectively. then (E/F, $\Phi(\eta),(\tau))$ is the structured $*$-inductive limit of $(E, V, \tau)$ with respect to $\Phi$.

EXAMPLE 2. Let $\left(E_{1}, \nabla_{1}, \tau_{1}\right)$ be a family of structured topological vector spaces. Let $E=\oplus E_{1} \subseteq I I E_{1}$ be the direct sum of the vector spaces $E_{1}$, with the structure $V$ induced by the product structure of $I I E_{1}$. The structure *-inductive limit topology on (E, $V$ ) with respect to the structured topological vector spaces $\left(E_{i}, V_{i}, \tau_{i}\right)$ and the structured homomorphisms $\left\{J_{i}\right\}$ (iEI) is called the structured *-direct sum topology on ( $E, V$ ).

Let $\left\{\left(E_{1}, \nabla_{1}, \tau_{1}\right)\right\}$ (iEI) be a family of structured topological vector spaces where $\nabla_{1}$ is the general linear structure (see $\$ 4$ example 6 ) on each $E_{1}$. Let (E, $V$ be a structured space with $\boldsymbol{V}$ the general linear structure on $E$, and suppose that $\left\{g_{1}\right\}$ is a family of structured homomorphisms $g_{i}: E_{1} \rightarrow E$ such that $E=\cup g_{1}$ $\left(E_{1}\right)$. Then the structured *-inductive limit topology on $(E, V)$ coincides with

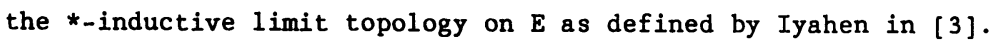

By modifying the structures (see $\$ 4$ ) $\nabla_{1}$ and $V$ in the obvious way, we can in a similar manner arrive at the notions of algebraic *-inductive limits and $0-{ }_{-}$ inductive limits discussed in [8], [7]. 
THEOREM 3.2 Let $\tau$ be the structured *-inductive limit topology on the structured space (E, $V$ ) with respect to the structured topological vector spaces $\left(E_{1}, V_{1}, \tau_{1}\right)(i \in I)$ and structured homomorphisms $\left\{g_{i}\right\}$. Then $V \in V$ is a $\tau-$ neighbourhood of $O$ in $E$ if and only if $V$ has a defining sequence $\left\{V_{n}\right\}$ of absorbing sets in $V$ such that $g_{1}^{-1}\left(V_{n}\right)$ is a $\tau_{1}$-neighbourhood of 0 in $E_{1}$ for $n=$ $1,2, \ldots$ and for each $i \in I$.

PROOF. This is similar to the proof of Theorem 3.2 of [8].

COROLLARY. If $f$ is a structured homomorphism from $E$ into any structured topological vector space $F$, then $f$ is continuous if and only if $f_{\circ} g_{1}$ is continuous for all ieI.

THEOREM 3.3. Let $E$ be the structured *-inductive limit of structured topological vector spaces $\left\{E_{i}\right\}$ ( $i \in I$ ) with respect to the structured homomorphisms $\left\{g_{1}\right\}$. If $E_{1}$ is $B_{1}$-ultrabornological for each $i \in I$, and $B$ is a family of bounded subsets of $E$ containing the family of subsets $\left\{g_{1}\left(B_{1}\right): B_{1} \in B_{1}, i \in I\right\}$ then $E$ is $B$ ultrabornological.

PROOF. Let $t$ be a $B$-bounded linear mapping from $E$ into any structured topological vector space $(F, W, \eta)$. For each $i \in I, g_{1}$ is continuous and so, by Proposition 2.7, is $B_{1}$-bounded. As $B$ contains the family $\left\{g_{i}\left(B_{i}\right): B_{i} \in B_{1} i \in I\right\}$ it is easy to check that $\operatorname{tog}_{1}$ is a $B_{1}$-bounded linear mapping from $E_{1}$ into $F$. Since $E_{1}$ is $B_{i}$-ultrabornological for each $i \in I$, by Corollary to Theorem 2.10, tog is continuous from $E_{1}$ into $F$ for each $i \in I$, and so it follows that $t$ is continuous by Corollary to Theorem 3.2. A repeated application of the Corollary to Theorem 3.2 shows that $E$ is B-ultrabornological.

\section{EXAYPLES}

We describe three different classes of structure which fit naturally with the general patterns we have developed here. First we consider convex structures; these (except 3 ) were introduced in the first instance by Warner in [10].

(1) Let $E$ be a vector space and let $C_{1}$ be the collection of all nonempty convex, balanced subsets of $\mathrm{E}$.

(2) Let $A$ be a linear associative algebra and let $C_{2}$ be the collection of all scalar multiples of all non-empty balanced, convex, idempotent subsets of $A$. With $A$ as above, let $C_{3}$ be the collection of all scalar multiples of all non-empty balanced, A-convex subsets of $A$.

(4) Let (E, C) be a partially ordered vector space and that $C_{4}$ be the collection of all non-empty balanced, convex and order-convex subsets of $\mathrm{E}$.

Let $(E, C)$ be a vector lattice and let $C_{5}$ be the collection of all non-empty convex, solid subsets of $E$.

In each of the above cases, $C_{1},(i=1,2,3,4,5)$ defines a structure on $E$ or $A$ as the case may be. If $A$ is a linear associative algebra $C_{2} \leq C_{3} \leq C_{1}$ and if $E$ is a vector lattice $C_{5} \subseteq C_{4} \subseteq C_{1} . C_{1}(i=1,2,3,4,5)$ are, respectively, called the convex linear structure, convex algebraic (iointly multiplicative) 
structure, convex algebraic (separately multiplicative) structure, convex order structure, and convex lattice structure. The topologies compatible with $C_{1}$ ( $i$ $=1,2,3,4,5)$ are respectively locally convex topologies, locally m-convex topologies (Michael [5]), locally A-convex topologies (Cochran et al [1]), locally o-convex topologies (Kist [4]), locally convex solid topologies (Wong and $\mathrm{Ng}$ [11]). The bornological properties of the structure $C_{1}(i=1,2,4,5)$ have been discussed by Warner [10] and $C_{3}$ by Cochran [2].

If in the collections of subsets given by $c_{i}(i=1,2,3,4,5)$ above, we replace convex by $p$-convex $(0<p<1)$ then we get the corresponding $p$-convex structures. These may be regarded as the straightforward generalisations of Warner's scheme.

We now introduce the notion of general structures on vector spaces and algebras.

(6) Let $E$ be a vector space and let $V_{1}$ be the collection of all nonempty balanced subsets $V$ of $E$ such that each $V$ has a defining sequence consisting of balanced sets. Then $\nabla_{1}$ defines a structure on $E$ which we call the general linear structure. Clearly $\nabla_{1}$ contains the collection of all suprabarrels in $E$. It follows that every linear topology on $E$ is compatible with $\nabla_{1}$. Let $\left(E, V_{1}, \tau\right)$ be a structured topological vector space with $\nabla_{1}$, the general linear structure. If $B$ is the class of all $\tau$-bounded subsets of $E$, then $E$ is B-ultrabornological if and only if $E$ is ultrabornological ([3],p.298). If $B=\phi$, then $E$ is $B$-ultrabornological if and only if the topology of $E$ is the finest linear topology on E. ([6]).

(7) Let $A$ be a linear associative algebra. The collection $\nabla_{2}$ of all non-empty balanced, idempotent subsets of $A$ each of which has a defining sequence of balanced, idempotent subsets, defines a structure on $A$. $\nabla_{2}$ is called the general algebraic structure on $A$. The family of all i-suprabarrels (see [8]) is a subset of $\nabla_{2}$ and so the topologies on $A$ compatible with $\nabla_{2}$ are precisely locally idempotent topologies on $A$ [8]. If $A$ is a locally idempotent algebra with the general algebraic structure and $B$ is the class of all i-bounded sets (see [9], p.197), then A is B-ultrabornological if and only if $A$ is i-ultrabornological ([8]). If $B$ is the null set, then $A$ is B-ultrabornological if and only if the topology of $A$ is the finest locally idempotent topology on A.

(8) Let $(E, C)$ be a partially ordered vector space. The collection $\nabla_{4}$ of all non-empty balanced, order-convex subsets $V$ of $E$ such that each $V$ has a defining sequence consisting of balanced order-convex subsets, is a structure on $E . \quad V_{4}$ is called the general order structure on $E$ determined by the given partial ordering. Every positive linear mapping from $E$ into any partially ordered vector space $F$ is a structured homomorphism with respect to the associated general order structures. Every o-suprabarrel of $\mathrm{E}$ belongs $\boldsymbol{V}_{4}$ (see [7]) and so the locally order-convex topologies on $E$ are compatible 


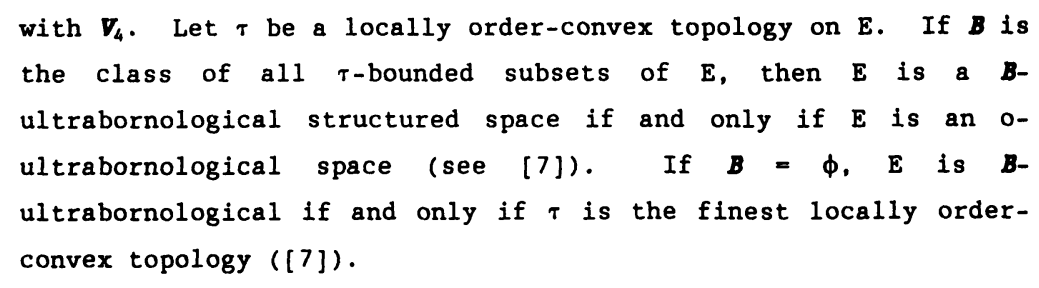

I wish to thank Dr $K$. Rowlands for his helpful comments and suggestions.

\section{REFERTICES}

1. Cochran, A.C., Williams, C.R. and Keown, R. "On a class of topological algebras," Pacific J. Math., 34, (1970), 17-25.

2. Cochran, A.C. "Inductive limits of A-convex algebras," Proc. Amer. Math. Soc., 37. (1973), 489-496.

3. Iyahen, S.0. "On a certain class of linear topological spaces," Proc. London Math. Soc., (3), 18, (1968), 285-307.

4. Kist, J. "Locally o-convex spaces," Duke. Math, J., 25, (1958), 569-582.

5. Michael, E.A. "Locally multiplicatively convex topological algebras," Mem. Amer. Math. Soc., 11, (1952).

6. Murali, V. "Suprabarrels in topological vector spaces," Math Japonica, $32(5),(1987), 797-800$.

7. Murali, V. "Locally order-convex spaces," Kyungpook Math. J., 18(1), (1978), 37-46.

8. Murali, V. :Locally idempotent algebras," Math Japonica, 30(5), (1985), 763-776.

9. Warner, S. "Inductive limits of normed algebras," Trans. Amer, Math. Soc., 82, (1956), 190-216.

10. Warner, S. "Bornological structures," Illinois J. Math., 4, (1960), 231245 .

11. Wong, $\mathrm{Y}$. and $\mathrm{Ng}, \mathrm{K}$. Partially ordered topological vector spaces," Oxford Math. Monographs, (1973). 


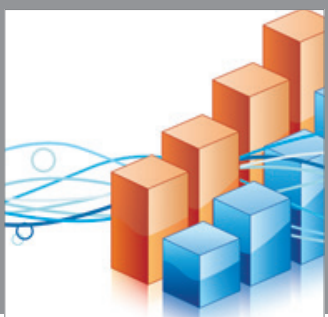

Advances in

Operations Research

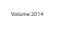

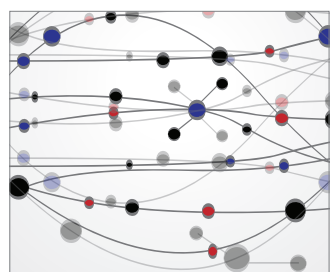

\section{The Scientific} World Journal
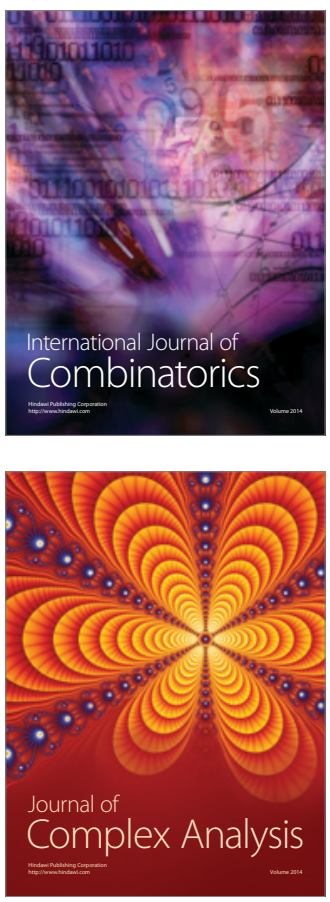

International Journal of

Mathematics and

Mathematical

Sciences
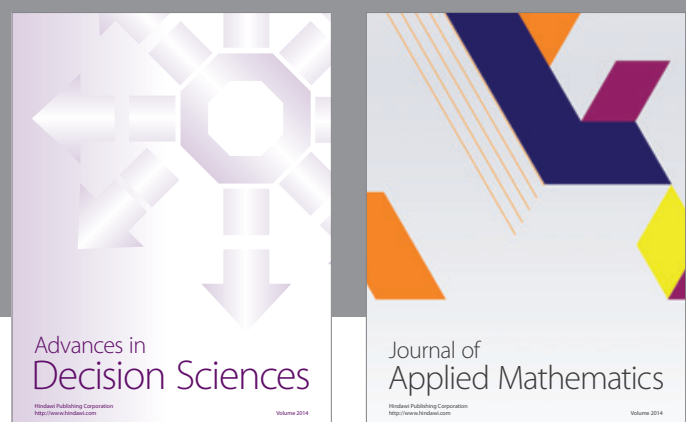

Journal of

Applied Mathematics
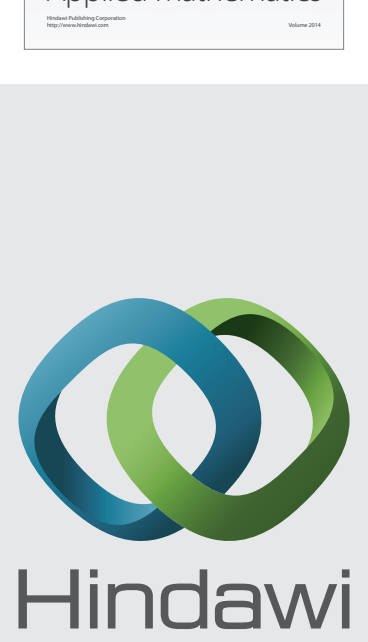

Submit your manuscripts at http://www.hindawi.com
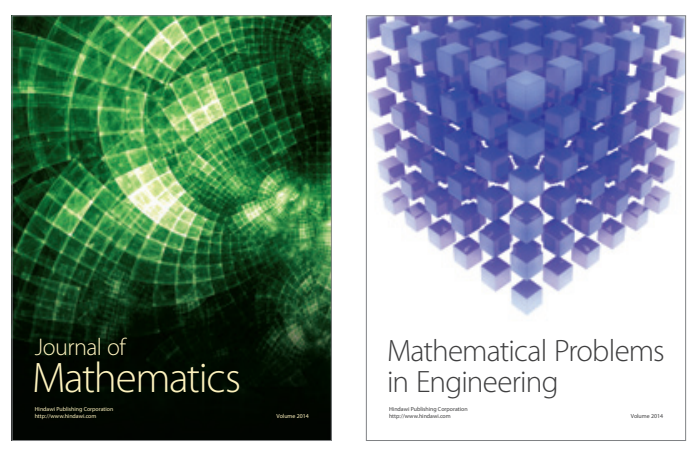

Mathematical Problems in Engineering
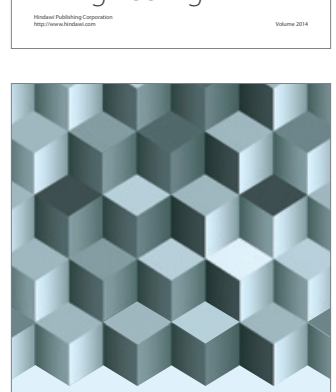

Journal of

Function Spaces
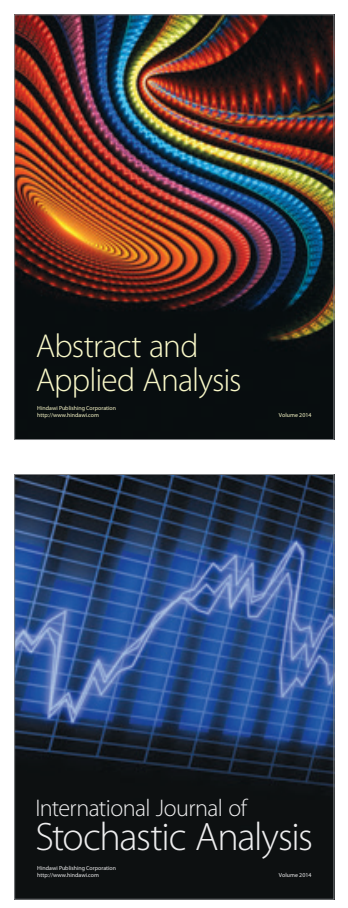

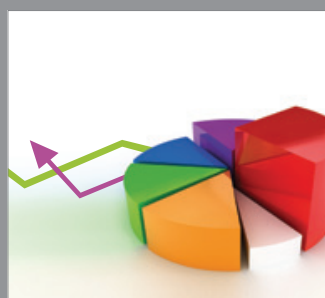

ournal of

Probability and Statistics

Promensencen
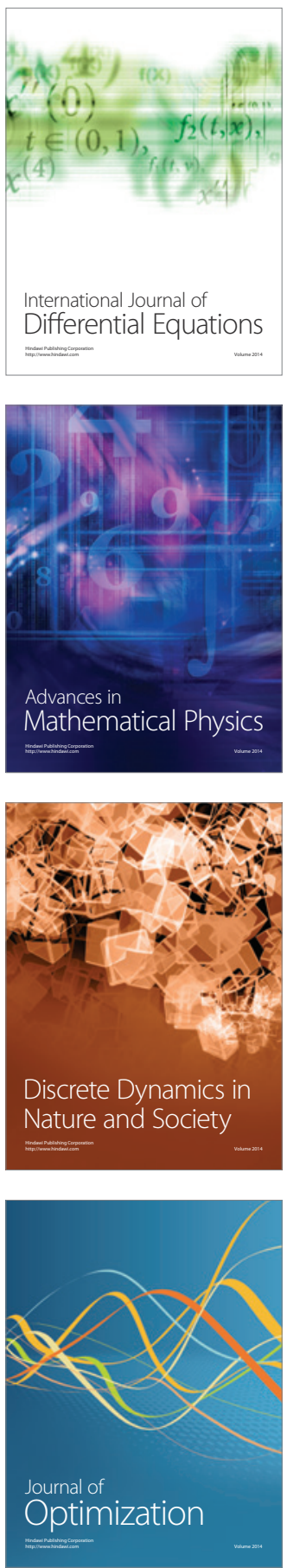